\title{
Abnormal Baseline liver function tests are associated with death or mechanical ventilation in COVID-19
}

\author{
Bashar Fteiha ${ }^{1}$, Haytham Abu Khdair ${ }^{1}$, Dolev Perez ${ }^{1}$, Hani Karameh ${ }^{1}$, Ron Skorochod $^{2}$, \\ Eran Goldin ${ }^{1}$, and Mahmud Mahamid ${ }^{1}$ \\ ${ }^{1}$ Shaare Zedek Medical Center \\ ${ }^{2}$ Hebrew University Hadassah Medical School
}

October 29, 2020

\begin{abstract}
Background - Studies investigating the relation between baseline liver abnormality and COVID-19 patients' outcomes during hospitalization are scarce. The aim of the study is to address and characterize this clinically important association. Methods

- Retrospective single-center study of adults hospitalized with COVID-19 infection for whom the baseline liver function tests up to one year prior to the admission were available. The study cohort included hospitalized patients from COVID-19 wards and specialized COVID-19 intensive care unit. Subjects were divided into a normal and abnormal baseline LFT groups that were then compared with respect to demographic characteristics, co-morbidities and patients' outcomes during hospitalization. Results - 133 of 444 subjects met the inclusion criteria and were included in the study. Of them, 50/133 (37.6\%) had abnormal baseline LFTs. The mean age of the cohort subjects was $65.7 \pm 22.1$ years and the mean BMI was $28.7 \pm 13.0$. Subjects with abnormal LFTs were more likely to die $(22 \%$ versus $4.8 \%, \mathrm{p}=0.004)$ or require mechanical ventilation $(16 \%$ versus $4.8 \%, \mathrm{p}=$ 0.03) during hospitalization when compared to their normal LFT counterparts. Multivariate analysis revealed that abnormal baseline LFT (OR 6, 95\% CI 2.0 - 18.4) was the strongest predictor of death or requiring mechanical ventilation followed by diabetes mellitus (OR 4.5, 95\% CI 1.3 - 14.8) and congestive heart failure (OR 3.9, 95\% CI 1.2 - 12.5). Conclusion - patients known to have a baseline LFTs abnormality appear to be at an increased risk for death or mechanical ventilation during hospitalization with COVID-19.
\end{abstract}

\section{Abnormal Baseline liver function tests are associated with death or mechanical ventilation in COVID-19}

Bashar Fteiha ${ }^{1}$, Haytham Abu Khdair ${ }^{2}$, Dolev Perez ${ }^{2}$, Hani Karameh², Ron Skorochod ${ }^{2}$, Eran Goldin ${ }^{1}$, Mahmud Mahamid ${ }^{1}$.

${ }^{1}$ Faculty of Medicine, Hebrew University of Jerusalem, Israel; Department of gastroenterology and liver diseases, Shaare Zedek Medical Center, Jerusalem 9103102, Israel.

${ }^{2}$ Faculty of Medicine, Hebrew University of Jerusalem, Israel; Internal Medicine Department, Shaare Zedek Medical Center, Jerusalem 9103102, Israel

Word count: 2362 words.

Total number of figures and tables: 2 Figures and 3 Tables.

Abbreviations - LFTs, liver function tests. AST, Aspartate transaminase. ALT, Alanine transaminase. ALP, Alkaline phosphatase, ASA, aspirin. BMI, body mass index. OR, odds ratio. MV, mechanical ventilation.

Conflict of interest - The authors states that they have no conflict of interest regarding this work. 
Funding - The authors did not receive support from any organization for the submitted work.

Corresponding Author: Bashar Fteiha, MD

Digestive Diseases Institute, Shaare Zedek Medical Center, Jerusalem, Israel

Email: basharf@szmc.org.il Tel: +9725298086

https://orcid.org/0000-0001-7676-9447

\section{Abstract}

Background - Studies investigating the relation between baseline liver abnormality and COVID-19 patients' outcomes during hospitalization are scarce. The aim of the study is to address and characterize this clinically important association.

Methods - Retrospective single-center study of adults hospitalized with COVID-19 infection for whom the baseline liver function tests up to one year prior to the admission were available. The study cohort included hospitalized patients from COVID-19 wards and specialized COVID-19 intensive care unit. Subjects were divided into a normal and abnormal baseline LFT groups that were then compared with respect to demographic characteristics, co-morbidities and patients' outcomes during hospitalization.

Results - 133 of 444 subjects met the inclusion criteria and were included in the study. Of them, 50/133 $(37.6 \%)$ had abnormal baseline LFTs. The mean age of the cohort subjects was $65.7 \pm 22.1$ years and the mean BMI was $28.7 \pm 13.0$. Subjects with abnormal LFTs were more likely to die $(22 \%$ versus $4.8 \%, \mathrm{p}=$ $0.004)$ or require mechanical ventilation $(16 \%$ versus $4.8 \%, \mathrm{p}=0.03)$ during hospitalization when compared to their normal LFT counterparts. Multivariate analysis revealed that abnormal baseline LFT (OR 6, 95\% CI 2.0 - 18.4) was the strongest predictor of death or requiring mechanical ventilation followed by diabetes mellitus (OR 4.5, 95\% CI 1.3 - 14.8) and congestive heart failure (OR 3.9, 95\% CI 1.2 - 12.5).

Conclusion - patients known to have a baseline LFTs abnormality appear to be at an increased risk for death or mechanical ventilation during hospitalization with COVID-19.

Keywords - COVID-19; Liver function tests; Death; mechanical ventilation.

\section{What's known?}

Abnormal Liver function tests (LFTs) are common in patients hospitalized with COVID-19 infection, especially in the intensive care unit.

LFTs abnormalities have been shown to correlate with different negative outcomes and poor prognosis in COVID-19 infection.

\section{What's new?}

Abnormal baseline liver function tests (LFTs) in patients with no pre-existing liver disease are independently associated with a higher chance of death or mechanical ventilation during hospitalization with COVID-19 infection.

Future research should strive to further characterize this clinically important association, in light of the increased adverse outcomes in this highly prevalent and susceptible risk group of patients with COVID-19 infection.

\section{Introduction}

The ongoing COVID-19 pandemic caused by the novel corona virus SARS-COV-2 remains a significant global public health threat. Ever since the isolation of SARS-COV-2 in a cluster of pneumonia cases from Wuhan in China ${ }^{1}$, it has spread globally at an alarming rate where it continues to overburden public health systems thus resulting in substantial morbidity and mortality. 
Abnormal liver function tests (LFTs) is a common finding among hospitalized COVID-19 patients and is being increasingly recognized as the most common extra-pulmonary manifestation of COVID-19 infection ${ }^{2}$. A recent meta-analysis concluded that abnormal aspartate aminotransferase (AST) and Alanine transaminase (ALT) were reported in $50 \%$ and $40 \%$ of patients admitted to the normal COVID-19 wards and both were quantitively higher in ICU-treated patients ${ }^{3}$. The LFTs abnormality in COVID-19 patients is not merely a manifestation of hepatic injury or the severity of the underlying illness, but may also bear a prognostic significance as these patients appear to have poorer outcomes compared to their counterparts with normal LFTs $^{4}$. Abnormal liver function tests during hospitalization were shown to be independently associated with death or transfer to ICU unit in a retrospective multi-centric Italian Cohort ${ }^{5}$, and patients with abnormal liver tests had significantly higher odds of developing severe pneumonia in a cross-sectional study conducted in China ${ }^{6}$.

Most of the existing literature have focused almost exclusively on the association between abnormal LFTs and patients' outcomes during hospitalization, with a relative scarcity of studies investigating the relation between baseline liver function abnormality and patient outcomes during hospitalization. We therefore designed and conducted a single-center observational study in an attempt to address this potential and clinically important association.

\section{Methods}

This was a retrospective cohort study conducted at the Sharee Zedek Medical Center (SZMC) University Hospital located in Jerusalem, Israel. We reviewed the hospital records of all consecutive adult patients hospitalized over a four-month span (March 2019 to June 2019) at SZMC. Included were adult patients ([?] 18 years) hospitalized for COVID-19 infection for whom the baseline liver function tests up to one year prior to the admission were available. Subjects who were less than 18 years of age and medical personnel or for whom the baseline liver function tests were not available were excluded. The study cohort included patients hospitalized in the specialized COVID-19 hospitalized wards and COVID-19 specialized intensive care unit. The diagnosis of COVID-19 infection was based on positive PCR nasopharyngeal swab specimen tested for SARS-CoV-2.

The current study received ethical approval from the local hospital ethical committee and was conducted according to the Helsinki declaration and its subsequent amendments. Data were coded in order to preserve the anonymity of the patients. Informed consent was waived because of the non-interventional study design.

\section{Data collection}

Epidemiological, clinical and laboratory data of all patients with laboratory-confirmed SARS-CoV-2 were obtained with data collection forms from the electronic medical records of each patient. The data collected included demographics, comorbidities, the use of any potentially hepatotoxic medications, and lab results available from a primary health care setting within the range of one year prior to admission. Upon their admission, the severity of all patients was recorded and their labs results and outcomes during hospitalization were extracted from the electronic medical file.

Patients with known hepatic pathology or autoimmune phenotypes (such as alcoholic liver disease, druginduced liver injury, autoimmune hepatitis, viral hepatitis, cholestatic liver disease and metabolic/genetic liver disease) were excluded using specific clinical, laboratory, radiological and/or histological criteria/tests (serology of viral hepatitis A, B, and C, autoimmune markers including ANA, anti-LKM, anti-smooth mussels protein electrophoresis, immune electrophoresis, metabolic markers such as serum ceruloplasmin, 24-h urine collection for copper, ferritin, iron, transferrin saturation, TSH, HbA1c and alpha-1 antitrypsin).

\section{Definitions}

Abnormal Liver function tests (LFT's) were defined as an elevation of at least one of AST, ALT, ALP, GGT or bilirubin above the upper limit of normal reference range of local laboratory ${ }^{7}$. The pattern of abnormal LFTs was defined as hepatocellular when patients presented with predominantly raised ALT and AST; cholestatic when patients showed predominantly raised ALP and GGT, and mixed when the extent 
of AST/ALT and ALP/GGT was similar ${ }^{8}$. COVID-19 severity of each patient was based on the most severe condition during the entire course of the disease, with the clinical classification based on the most recently adopted tentative $8^{\text {th }}$ edition protocol for diagnosis and treatment for COVID-19 patients, released by the national health commission of China ${ }^{9}$. The primary outcome measure was defined as the composite endpoint of death or requirement for mechanical ventilation during hospitalization.

\section{Statistical analysis}

Before commencing any statistical processing and analysis, data were visually inspected and checked for outliers. The analysis was performed using the commercial software Statistical Package for Social Science (SPSS version 24.0, IBM, Chicago, IL, USA). Categorial variables were tested using the chi-square test or Fisher's exact test, as appropriate. Continuous variables were examined using the student's t test if normally distributed and Man-Whitney test if not. To identify variables associated with the primary outcome measure, univariate analysis was performed. Variables that were significantly associated $(\mathrm{p}<0.05)$ with the primary outcome measure (defined as the composite endpoint of death or requirement for mechanical ventilation during hospitalization) were entered into the multivariate logistic regression model in a stepwise fashion. Adjusted odds-ratios (ORs) and 95\% confidence intervals (CIs) for the likelihood of occurrence of primary outcome measure were calculated. Statistical significance was set at p-value less than 0.05 .

\section{Results}

Between the $9^{\text {th }}$ of March and the $10^{\text {th }}$ of June, 444 patients were hospitalized in the COVID-19 treatment wards (including a specialized COVID-19 care ICU). After the application of exclusion criteria (lack of baseline LFTs in previous year, lack of medical information in medical file or history of known hepatic disease), the final study cohort consisted of 133 patients (figure 1).

The characteristics of the study cohort are outlined in table 1 below. The mean age of the cohort subjects was $65.7+-22.1$ years and the mean BMI was $28.7+-13.0$. Seventy-three were males $(54.9 \%)$. The most frequently reported co-morbidities were Dyslipidemia and hypertension (64.6\% and $45.1 \%$, respectively). The mean baseline AST and ALT levels were

$28.4+-21.9$ and $25.7+-30.2$, respectively. The most commonly used chronic medications were ASA (15\%) and statins $(36.1 \%)$.

Subjects in the study cohort were then classified based on their LFTs into 2 groups; a group with abnormal baseline LFTs that consisted on 50 subjects and a group of normal baselines LFTs that included 83 patients. The two groups were then compared with respect to their demographic, clinical characteristics, mean LFTs values and outcomes observed during hospitalization. No statistically significant differences were noted between the two groups with respect to baseline demographic and co-morbidities, but subjects with abnormal LFTs were more likely to die $(22 \%$ versus $4.8 \%, \mathrm{p}=0.004)$ or require mechanical ventilation ( $16 \%$ versus $4.8 \%, \mathrm{p}=0.03$ ) during hospitalization when compared to their normal LFT counterparts. (Table 2).

Relation between the pattern of liver injury $\&$ patients' outcomes - In order to characterize the relation between specific patterns of liver injury and adverse outcomes, subjects were abnormal LFTs group were broken down into two subgroups (hepatocellular and cholestatic) depending on the predominantly elevated hepatic dysfunction marker. Comparison between these different patterns of liver injury revealed that patients with predominantly cholestatic pattern of injury were more likely to meet the primary composite outcome compared to those with predominant hepatocellular injury (Figure 2).

Does abnormal baseline LFTs independently predict death or mechanical ventilation during hospitalization with COVID-19 infection?

A multivariate logistic regression was then performed to ascertain the effects of age, different background illnesses and abnormal baseline LFTS on the primary outcome measure - composite endpoint of death or requirement for mechanical ventilation during hospitalization. Results are presented in table (3) below. 
During the 4-month span of the study and out of the 444 subjects hospitalized with COVID-19 in our tertiary care center, $32(7.2 \%)$ patients died and $21(4.7 \%)$ required mechanical ventilation. The overall composite endpoint was identified in $49(11 \%)$ subjects. Univariate analysis revealed that age above 65 years, Diabetes Mellitus, Hypertension, congestive heart failure, and abnormal baseline LFTs were all were independently associated with the composite endpoint. Multivariate analysis revealed that abnormal baseline LFTs (OR $6,95 \%$ CI $2.0-18.4)$ was the strongest predictor of meeting the composite endpoint followed by diabetes mellitus (OR 4.5, 95\% CI $1.3-14.8$ ) and congestive heart failure (OR 3.9, 95\% CI $1.2-12.5$ ).

\section{Discussion}

In our study, patients with elevated baseline LFTs had a higher chance of meeting the composite endpoint of death or mechanical ventilation during hospitalization. To the best of our knowledge, this is the first study to document the relation between abnormal baseline LFTs and the susceptibility to a more severe COVID-19 course during hospitalization.

Most of the studies that looked into the association between COVID-19 and the liver have either described the pattern of liver injury due to COVID-19 or the clinical characteristics and outcomes of patients presenting with hepatic dysfunction due to SARS-COV-2 $2^{2,4,10,11}$. The ubiquitous distribution of SARS-COV-2 receptor for viral entry, ACE2, on Cholangiocytes naturally points towards a hepatic injury being a common systemic manifestation of extrapulmonary COVID-19 infection ${ }^{12}$. Possible mechanisms that may potentially underlie this association include immune-mediated damage as a result of the severe inflammatory response commonly referred to as "the cytokine storm" ${ }^{13}$, direct hepatotoxicity due to viral replication in hepatic cells, anoxic damage (hypoxic hepatitis) that may accompany respiratory failure, Drug-induced liver injury (DILI) and the reactivation of a pre-existing liver disease ${ }^{14-16}$.

Given the heterogenous nature of different liver diseases, the outcomes of patients with pre-existing liver disease that become infected with COVID-19 may vary significantly depending on the underlying liver disease, presumably due to a modifying element of immunosuppression on the inflammatory response that predominates the illness. As the host inflammatory response appears to be the main driver of pulmonary damage in this infection,

a useful framework to characterize this association would be to divide the pre-existing liver diseases into 2 categories; those with an attenuated inflammatory response and those with pronounced inflammatory responses. Examples of the former category include patients with autoimmune hepatitis, malignancies, and liver transplant recipients where preliminary evidence suggests that these patients might not be at an increased risk of severe complications compared to the general population ${ }^{17}$. On the other hand, patients with chronic liver disease that are associated with hyperinflammatory states such as non-alcoholic fatty liver disease (NAFLD) and cirrhosis appear to be at an increased risk of severe COVID-19 course ${ }^{18,19}$. NAFLD patients in particular appear to be at an increased risk for developing hepatic injury during hospitalization due to the deleterious interplay of chronically active inflammatory pathways and the acute cytokine storm that accompanies COVID-1920. Often referred to as the hepatic manifestation of metabolic syndrome, NAFLD was shown to be an independent risk factor for severe COVID-19 infection even in the absence of other constituents of metabolic syndrome ${ }^{21}$.

Our study has several limitations; namely the inherent limitations of relatively small sample size and retrospective, single-center design but it highlights a clinically important association and emphasizes the unmet need for larger scale studies to further characterize the relation between liver disease and COVID-19 clinical course. The exact cause of pre-existing liver conditions has not been outlined in the majority of included subjects, which makes it difficult to analyze the impact of COVID-19 on the different etiologies of pre-existing liver diseases.

No correlation was made between the degree of hepatic dysfunction with inflammatory markers or radiological findings during hospitalization, which could have allowed for a better understanding of the association between abnormal LFTs and MV or mortality. 
Finally, the high prevalence of hyperlipidemia and obesity in our cohort may have contributed to an overestimation of NAFLD impact on patients' outcomes while underestimating other pre-existing liver diseases impact, although this is representative of larger COVID-19 patient cohorts where obesity and other metabolic syndrome appear frequently among the risk factors and are thought to be harbingers of adverse clinical outcomes.

In conclusion, patients known to have a baseline LFTs abnormality appear to be at an increased risk for death or mechanical ventilation during hospitalization with COVID-19 infection. Further large scale preferably prospective studies are urgently needed to characterize this clinically important association.

\section{Conflict of interest statement}

The authors declare they have no conflict of interest.

\section{Author contributions}

Bashar Fteiha - Study conception and design, Collection of data, analysis and interpretation of results, drafting the manuscript.

Haitham Abu Khdair - Collection of data, analysis and interpretation of results, drafting the manuscript.

Dolev Perez - Collection of data.

Hani Karameh - Collection of data.

Ron Skorochod - Collection of data.

Batsheva Ziff-Werman - Collection of data.

Itamar Feldman - Collection of data.

Itamar Feldman - Collection of data.

Alon Bnaya - Collection of data.

Eran Goldin - Analysis and interpretation of data, critical revision.

Mahmud Mahamid - Study conception and design, drafting of manuscript and critical revision.

\section{References:}

1. Zhu N, Zhang D, Wang W, Li X, Yang B, Song J et al. A Novel Coronavirus from Patients with Pneumonia in China, 2019. N Engl J Med 2020;382:727-33.

2. Xu L, Liu J, Lu M, Yang D, Zheng X. Liver injury during highly pathogenic human coronavirus infections. Liver Int 2020;40:998-1004.

3. Labenz C, Toenges G, Worns M-A, Sprinzl MF, Galle PR, Schattenberg JM. Liver injury in patients with severe acute respiratory syndrome coronavirus-2 infection: a systematic review and meta-analysis. European Journal of Gastroenterology \& Hepatology 9000;Publish Ahead of Print.

4. Xie H, Zhao J, Lian N, Lin S, Xie Q, Zhuo H. Clinical characteristics of non-ICU hospitalized patients with coronavirus disease 2019 and liver injury: A retrospective study. Liver Int 2020;40:1321-6.

5. Piano S, Dalbeni A, Vettore E, Benfaremo D, Mattioli M, Gambino CG et al. Abnormal liver function tests predict transfer to intensive care unit and death in COVID-19. Liver Int 2020.

6. Cai Q, Huang D, Yu H, Zhu Z, Xia Z, Su Y et al. COVID-19: Abnormal liver function tests. J Hepatol 2020;73:566-74.

7. Newsome PN, Cramb R, Davison SM, Dillon JF, Foulerton M, Godfrey EM et al. Guidelines on the management of abnormal liver blood tests. Gut 2018;67:6-19. 
8. Musana KA, Yale SH, Abdulkarim AS. Tests of liver injury. Clin Med Res 2004;2:129-31.

9. China NHCoTPsRo. Diagnosis and Treatment Protocol for COVID-19 Patients (Tentative 8th Edition). 2020 .

10. Jiang S, Wang R, Li L, Hong D, Ru R, Rao Y et al. Liver Injury in Critically Ill and Non-critically Ill COVID-19 Patients: A Multicenter, Retrospective, Observational Study. Front Med (Lausanne) 2020;7:347.

11. Bertolini A, van de Peppel IP, Bodewes F, Moshage H, Fantin A, Farinati F et al. Abnormal liver function tests in COVID-19 patients: relevance and potential pathogenesis. Hepatology 2020.

12. Zhang C, Shi L, Wang FS. Liver injury in COVID-19: management and challenges. Lancet Gastroenterol Hepatol 2020;5:428-30.

13. Mehta P, McAuley DF, Brown M, Sanchez E, Tattersall RS, Manson JJ et al. COVID-19: consider cytokine storm syndromes and immunosuppression. Lancet 2020;395:1033-4.

14. Muhovic D, Bojovic J, Bulatovic A, Vukcevic B, Ratkovic M, Lazovic R et al. First case of drug-induced liver injury associated with the use of tocilizumab in a patient with COVID-19. Liver Int 2020.

15. Mantovani A, Beatrice G, Dalbeni A. Coronavirus disease 2019 and prevalence of chronic liver disease: A meta-analysis. Liver Int 2020;40:1316-20.

16. Napodano C, Pocino K, Stefanile A, Marino M, Miele L, Gulli F et al. COVID-19 and hepatic involvement: The liver as a main actor of the pandemic novel. Scand J Immunol 2020:e12977.

17. D'Antiga L. Coronaviruses and Immunosuppressed Patients: The Facts During the Third Epidemic. Liver Transpl 2020;26:832-4.

18. Qi X, Wang J, Li X, Wang Z, Liu Y, Yang H et al. Clinical course of COVID-19 in patients with pre-existing decompensated cirrhosis: initial report from China. Hepatol Int 2020;14:478-82.

19. Sharma P, Kumar A. Metabolic dysfunction associated fatty liver disease increases risk of severe Covid19. Diabetes Metab Syndr 2020;14:825-7.

20. Portincasa P, Krawczyk M, Smyk W, Lammert F, Di Ciaula A. COVID-19 and non-alcoholic fatty liver disease: Two intersecting pandemics. Eur J Clin Invest 2020;50:e13338.

21. Mahamid M, Nseir W, Khoury T, Mahamid B, Nubania A, Sub-Laban K et al. Nonalcoholic fatty liver disease is associated with COVID-19 severity independently of metabolic syndrome: a retrospective case-control study. Eur J Gastroenterol Hepatol 2020.

Tables \& Figures

Table 1. Demographic, clinical and lab characteristics of 133 subjects included in each of the study groups.

\begin{tabular}{ll}
\hline Characteristic & Total N $=133$ \\
\hline Age (years), mean \pm SD & $65.7 \pm 22.1$ \\
BMI - mean \pm SD & $28.7 \pm 13.0$ \\
Male Gender, n (\%) & $73(54.9)$ \\
Diabetes Mellitus, n (\%) & $42(31.6)$ \\
HTN, n (\%) & $60(45.1)$ \\
CHF, n (\%) & $22(16.5)$ \\
Dyslipidemia, n (\%) & $62(64.6)$ \\
Obesity, n (\%) & $51(38.3)$ \\
ASA treatment, n (\%) & $20(15)$ \\
Statin treatment, n (\%) & $48(36.1)$
\end{tabular}




\begin{tabular}{ll}
\hline Characteristic & Total N $=133$ \\
\hline Baseline AST U/L, Mean \pm SD & $28.4 \pm 21.9$ \\
Baseline ALT U/L, Mean \pm SD & $25.7 \pm 30.2$ \\
Baseline ALP U/L, Mean \pm SD & $93.5 \pm 55.8$ \\
GGT U/L, Mean \pm SD & $43.6 \pm 55.6$ \\
Total billirubin mg/dl, Mean \pm SD & $0.67 \pm 0.95$ \\
Direct Billirubin mg/dl, Mean \pm SD & $0.17 \pm 0.17$ \\
Albumin g/dl, Mean \pm SD & $4.2 \pm 0.62$ \\
Total protein g/dl, Mean \pm SD & $6.8 \pm 0.72$ \\
INR, Mean \pm SD & $1.05 \pm 0.22$ \\
\hline
\end{tabular}

Abbreviations - BMI, body mass index. HTN, hypertension. CHF, Congestive heart failure. ASA, aspirin. AST, Aspartate transaminase. ALT, Alanine transaminase. ALP, Alkaline phosphatase. GGT - gammaglutamyl transferase. INR - international normalized ratio. SD, standard deviation.

Table 2. Comparison between subjects with normal \& abnormal baseline LFTs.

\begin{tabular}{|c|c|c|c|c|}
\hline Characteristic & Total $\mathrm{N}=133$ & $\begin{array}{l}\text { Abnormal LFTs N } \\
=50\end{array}$ & $\begin{array}{l}\text { Normal LFTS N = } \\
83\end{array}$ & $\mathrm{P}$-value \\
\hline $\begin{array}{l}\text { Age (years), mean } \\
\pm \mathrm{SD}\end{array}$ & $65.7 \pm 22.1$ & $63.8 \pm 27.1$ & $66.9 \pm 18.6$ & 0.43 \\
\hline $\begin{array}{l}\mathrm{BMI}-\text { mean } \pm \\
\mathrm{SD}\end{array}$ & $28.7 \pm 13.0$ & $28.5 \pm 7.2$ & $28.9 \pm 15.3$ & 0.88 \\
\hline $\begin{array}{l}\text { Male Gender, } \mathrm{n} \\
(\%)\end{array}$ & $73(54.9)$ & $28(56)$ & $45(54.2)$ & 0.84 \\
\hline $\begin{array}{l}\text { Diabetes Mellitus, } \\
\mathrm{n}(\%)\end{array}$ & $42(31.6)$ & $15(30)$ & $27(32.5)$ & 0.76 \\
\hline HTN, n (\%) & $60(45.1)$ & $23(46)$ & $37(44.6)$ & 0.87 \\
\hline $\mathrm{CHF}, \mathrm{n}(\%)$ & $22(16.5)$ & $11(22)$ & $11(13.3)$ & 0.188 \\
\hline $\begin{array}{l}\text { Dyslipidemia, } \mathrm{n} \\
(\%)\end{array}$ & $62(64.6)$ & $22(44)$ & $40(48.2)$ & 0.63 \\
\hline Obesity, n (\%) & $51(38.3)$ & $22(44)$ & $29(34.9)$ & 0.29 \\
\hline $\begin{array}{l}\text { ASA treatment, n } \\
(\%)\end{array}$ & $20(15)$ & $9(18)$ & $11(13.3)$ & 0.45 \\
\hline $\begin{array}{l}\text { Statin treatment, } \\
\mathrm{n}(\%)\end{array}$ & $38(36.1)$ & $17(34.0)$ & $31(37.3)$ & 0.69 \\
\hline $\begin{array}{l}\text { MV requirement, } \\
\mathrm{n}(\%)\end{array}$ & $12(9)$ & $8(16)$ & $4(4.8)$ & 0.03 \\
\hline $\begin{array}{l}\text { Death during } \\
\text { hospitalization, } \mathrm{n} \\
(\%)\end{array}$ & $15(11.3)$ & $11(22)$ & $4(4.8)$ & 0.004 \\
\hline
\end{tabular}

Abbreviations - BMI, body mass index. HTN, hypertension. CHF, Congestive heart failure. ASA, aspirin. AST, Aspartate transaminase. ALT, Alanine transaminase. ALP, Alkaline phosphatase. GGT - gammaglutamyl transferase. INR - international normalized ratio. SD, standard deviation. ASA, amino-salicylic acid. MV, mechanical ventilation

Univariate analysis

Variable

OR (95\% CI) 


\begin{tabular}{ll}
\hline & Univariate analysis \\
\hline Age $>65$ & $2.5(0.6-9.5)$ \\
DM & $4.7(1.4-15.9)$ \\
CHF & $3.8(1.1-12.7)$ \\
HTN Dyslipidemia Obesity LFTs abnormality ASA use Statins use & $1.54(0.38-6) 0.99(0.24-4.0) 0.97(0.3-3.0) 6.3$ \\
\hline
\end{tabular}

Table 3. Univariate analysis and adjusted multivariate regression analysis of variables associated with the primary outcome measure; defined as death or requirement for mechanical ventilation during hospitalization.

LFTs abnormality - defined as an elevation of at least one of AST, ALT, ALP, GGT or bilirubin above the upper limit of normal reference range of local laboratory.

DM, diabetes mellitus. CHF, congestive heart failure. HTN, hypertension. LFTs - liver function tests. ASA $=$ aspirin.

Figure (1) - Flowchart of the study cohort.

Abbreviations - LFTs - liver function tests. ICU, intensive care unit.

Figure (2) - the relation between the pattern of liver injury and death, Mechanical ventilation (MV) or either. Abbreviations - MV, mechanical ventilation

\section{Hosted file}

Figure 1.pdf available at https://authorea.com/users/334336/articles/489662-abnormalbaseline-liver-function-tests-are-associated-with-death-or-mechanical-ventilation-incovid-19

\section{Hosted file}

Figure 2.pdf available at https://authorea.com/users/334336/articles/489662-abnormalbaseline-liver-function-tests-are-associated-with-death-or-mechanical-ventilation-incovid-19 